\title{
Novel allelic variants and evidence for a prevalent mutation in URAT1 causing renal hypouricemia: biochemical, genetics and functional analysis
}

\author{
Blanka Stiburkova ${ }^{\star, 1}$, Ivan Sebesta ${ }^{1,2}$, Kimiyoshi Ichida ${ }^{3,4}$, Makiko Nakamura ${ }^{3}$, Helena Hulkova ${ }^{1}$, \\ Vladimir Krylov $^{1,5}$, Lenka Kryspinova ${ }^{1}$ and Helena Jahnova ${ }^{1,6}$
}

Renal hypouricemia (RHUC) is a heterogeneous inherited disorder characterized by impaired tubular uric acid (UA) transport with severe complications, such as acute kidney injury (AKI). Type 1 is caused by a loss-of-function mutation in the SLC22A12 gene (URAT1), type 2 in the SLC2A9 gene (GLUT9). This article describes three Czech families with RHUC type 1. The serum UA in the probands was $0.9,1.1$ and $0.5 \mathrm{mg} / \mathrm{dl}$ and expressed as an increase in the fractional excretion of UA ( 48,43 and $39 \%$ ). The sequencing analysis of SLC22A12 revealed three novel variants: p.G366R, p.T467M and a deletion

p.L415_G417del. A detailed metabolic investigation in proband C for progressive visual failure supported suspicion of neuronal ceroid lipofuscinosis type 7 conditioned by the mutation in the MFSD8 gene. Functional studies showed significantly decreased urate uptake and a mis-localized URAT1 signal in p.G366R, p.L415_G417del and p.T467M. Furthermore, colocalization studies showed accumulation of URAT1 protein in the endoplasmic reticulum. The findings suggest that loss-of-function mutations cause RHUC via loss of UA absorption partly by protein misfolding. However, they do not necessarily lead to AKI and a possible genotype-phenotype correlation was not proposed. Furthermore, results confirm an uneven geographical and ethnic distribution of SLC22A12 variants; the p.L415_G417del mutation predominates in the Roma ethnic group in the Czech Republic.

European Journal of Human Genetics (2013) 21, 1067-1073; doi:10.1038/ejhg.2013.3; published online 6 February 2013

Keywords: renal hypouricemia; SLC22A12; URAT1; uric acid transporters

\section{INTRODUCTION}

Uric acid (UA) is the end product of purine metabolism in humans. UA is a potent antioxidant, maintains blood pressure under low salt conditions and may help arrest inflammatory demyelinating diseases such as optic neuritis linked to multiple sclerosis, through scavenging of peroxynitrite in the central nervous system. ${ }^{1,2}$ Transport mechanisms for urate are located in the proximal tubules, where urate is secreted and extensively reabsorbed. As a result the ratio of excreted urate is only about $10 \%$.

Renal hypouricemia (RHUC) is a heterogeneous inherited disorder characterized by impaired tubular UA transport, reabsorption insufficiency and/or acceleration of secretion with severe complications, such as acute kidney injury (AKI), renal failure and nephrolithiasis. Unfortunately, no treatment is available. Low UA concentrations in serum and its increased fractional excretion (FE-UA) can be used as preliminary biochemical diagnostic markers. An additional method for diagnosis confirmation and/or identification of the type of RHUC is molecular analysis of the major urate transporters, $S L C 22 A 12^{3}$ and SLC2A9. ${ }^{4}$ So far, $>100$ patients with a loss-of-function mutation (compound heterozygous and/or homozygous) in the SLC22A12 gene have been found with most of the described patients being Japanese (OMIM no. 220150, RHUC1). ${ }^{3,5-8}$ More than 10 patients with RHUC caused by heterozygous defects in the SLC2A9 gene, coding GLUT9, have been described in Japan (OMIM no. 612076, RHUC2). ${ }^{9,10}$ Homozygous and/or compound heterozygous loss-of-function mutations in SLC2A9 responsible for severe hypouricemia, and in most patients complicated by nephrolithiasis and AKI have been described in a variety of ethnic groups: Israeli-Arab, Ashkenazi-Jewish, Japanese, Czech and United Kingdom. ${ }^{11-14}$

The SLC22A12 gene is localized on chromosome 11q13. Ten exons encode two transcript variants of the URAT1 transporter (332 and 553 amino acids), which are specifically expressed on the apical membrane of the proximal tubules in the kidneys and in human vascular smooth muscle cells. ${ }^{3}$ Currently, 34 mutations in the SLC22A12 coding region (28 missense/nonsense, one splicing, two regulatory, one small deletion, one small insertion, one gross deletion) have been described worldwide. Twenty-five mutations are disease causing. ${ }^{3,5-8,15-19}$ Five URAT1 variants have been described with a gout phenotype, ${ }^{20}$ one variant is associated with increased FE-UA and two variants are associated with reduced FE-UA. ${ }^{21,22}$

\footnotetext{
${ }^{1}$ Institute of Inherited Metabolic Disorders, First Faculty of Medicine, Charles University in Prague and General University Hospital in Prague, Prague, Czech Republic; ${ }^{2}$ Institute of Medical Biochemistry and Laboratory Medicine, First Faculty of Medicine, Charles University in Prague and General University Hospital in Prague, Prague, Czech Republic; ${ }^{3}$ Department of Pathophysiology, Tokyo University of Pharmacy and Life Sciences, Tokyo, Japan; ${ }^{4}$ Division of Kidney and Hypertension, Jikei University School of Medicine, Tokyo, Japan; ${ }^{5}$ Charles University in Prague, Faculty of Science, Department of Cell Biology, Prague, Czech Republic; ${ }^{6}$ Department of Pediatrics, Third Faculty of Medicine, Charles University in Prague and Faculty Hospital Kralovske Vinohrady in Prague, Prague, Czech Republic

${ }^{*}$ Correspondence: Dr B Stiburkova, Institute of Inherited Metabolic Disorders, First Faculty of Medicine, Charles University in Prague and General University Hospital, Ke Karlovu 2, Prague 2128 53, Czech Republic. Tel: +420 224967680; Fax: + 420 224967119; E-mail: blanka.stiburkova@|f1.cuni.cz

Received 31 October 2012; revised 14 December 2012; accepted 2 January 2013; published online 6 February 2013
} 
In this article we present clinical, biochemical, molecular genetics and functional characterization of previously reported p.R477H (CM042475 SNP, source HGMD-PUBLIC 2012.1) and novel p.G366R, p.L415_G417del and p.T467M variants in SLC22A12 gene responsible for RHUC1 in three Czech families. Hereditary RHUC is a rare disorder, but should be considered in patients with unexplained hypouricemia.The part of the patients are asymptomatic, but some may experience urolithiasis and/or be predisposed to exercise-induced acute renal failure.

\section{MATERIALS AND METHODS}

\section{Biochemical analyses}

Creatinine in plasma and urine; UA in serum and urine; hypoxanthine and xanthine in urine and plasma; and western blotting of uromodulin was measured as reported by authors previously. ${ }^{23}$

\section{PCR amplification of SLC22A12 and sequence analysis}

The genomic DNA for PCR analysis was isolated from blood samples using a QIAamp DNA Blood Mini Kit (Qiagen GmbH, Hilden, Germany). The promoter region and all exons were amplified using PCR and sequenced directly as was reported previously. ${ }^{13}$ The reference genomic sequence was defined as version NC_000011.8, region 64114688..64126396. The reference protein sequence was defined as Q96S37 (http://www.uniprot.org/uniprot).

\section{Restriction enzyme analysis}

Sequence variants were analyzed in our control cohort of 300 alleles (75 males, 75 females, randomly selected among patients of the General University Hospital, Prague) using PCR RFLP. Ten microliters of the PCR reaction mixture was digested and $20 \mu \mathrm{l}$ of the digestion reaction mixture was analyzed using a $2 \%$ agarose gel in $1 \mathrm{x}$ TAE buffer. Using Ssi (Fermentas, Vilnius, Lithuania), wild-type (wt) exon 7 ( $425 \mathrm{bp}$ ) and c.1096 G >C were digested into five and six segments, respectively, (wt-219, 181, 14, 8 and $3 \mathrm{bp}$; c1096G >C 181, 117, 102, 14, 8 and 3 bp). BanII (New England BioLabs, Inc., Ipswich, MA, USA) digested wt exon 7 into two segments (268 and $157 \mathrm{bp}$ ). In the deletion variant c.1245_1253del, the BanII restriction site was absent. Using BceAI (New England BioLabs Inc.) wt exon 9 (384bp) was digested into two segments (290 and $94 \mathrm{bp}$ ) and c.1400C $>\mathrm{T}$ remained in one piece $\mathrm{NcoI}$ (Fermentas) cut wt exon 9 into two (232 and $152 \mathrm{bp}$ ) and c.1430 G > A into three $(232,107$ and $45 \mathrm{bp})$ segments.

\section{Functional studies of URAT1 variants}

SLC22A12 allelic variants were prepared from the wt (GeneBank AB071863) using a Gene Tailor Site-Directed Mutagenesis Kit (Invitrogen, Carlsbad, CA, USA) and cloned into pcDNA3 vector. Capped cRNAs were synthetized using a T7 mMESSAGE mMACHINE kit (Life Technologies Japan Ltd., Tokyo, Japan). Xenopus laevis oocytes were defolliculated using Collagenase $1 \mathrm{~A}$, and repeatedly washed in OR-2 solution containing $82 \mathrm{~mm} \mathrm{NaCl}, 5 \mathrm{~mm} \mathrm{KCl}, 1 \mathrm{~mm}$ $\mathrm{MgCl}_{2}, 5 \mathrm{~mm}$ HEPES, pH 7.6). Selected fully grown oocytes were then transferred to the injection and culture medium ND-96 (96 mM NaCl, $2 \mathrm{~mm}$ $\mathrm{KCl}, 1.8 \mathrm{mM} \mathrm{CaCl}_{2}, 5 \mathrm{~mm} \mathrm{MgCl}_{2}, 5 \mathrm{~mm}$ sodium pyruvate, $\mathrm{pH} 7.6$, penicillin $(10000 \mathrm{U} / \mathrm{ml})$ and streptomycin $(10 \mathrm{mg} / \mathrm{ml})$. In all, 18-20 oocytes were injected with $50 \mathrm{ng}$ of a particular cRNA using a Narishige IM300 pneumatic microinjector (Narishige Ltd., Tokyo, Japan) and then kept in ND-96 at $17^{\circ} \mathrm{C}$ for 2 days until subsequent immunocytochemical analysis or functional studies with radioactively labeled urate.

Uptake studies were performed as described previously with minor changes. ${ }^{10,18,24}$ Oocyte solubilization was done in $500 \mu \mathrm{l}$ of $0.0625 \mathrm{~N} \mathrm{NaOH}$ and final suspension was mixed with $3 \mathrm{ml}$ of ULTIMA GOLD (PerkinElmer, Inc., Waltham, MA, USA) for radioactivity determination using a scintillation counter (LSC-5100; ALOKA, Tokyo, Japan). Eight oocytes were used per experiment with tests repeated three times.

The subcellular localization of URAT1 mutants was determined using immunocytochemical analysis. Xenopus oocytes were fixed with $4 \%$ buffered paraformaldehyde in PBS overnight at room temperature. Immunodetection of URAT1 was performed on $3 \mu \mathrm{m}$ paraffin sections using rabbit anti-
SLC22A12 polyclonal antibody (Sigma, St Louis, MO, USA). Immunodetection of endoplasmic reticulum was performed using mouse anti-PDI monoclonal antibody (Enzo Life Sciences, Plymouth Meeting, PA, USA). The paraffin sections were stained after heat-induced antigen retrieval $(10 \mathrm{~mm}$ citrate buffer, $\mathrm{pH} 6.1$, for $20 \mathrm{~min}$ at $95.5^{\circ} \mathrm{C}$ in a water base) using standard blocking procedures. The primary antibody against URAT1 was applied diluted 1:25 in 5\% fetal bovine serum in PBS overnight at $4{ }^{\circ} \mathrm{C}$. Detection of bound primary antibodies were achieved using Alexa Fluor 488-conjugated with anti-rabbit IgG and Alexa Fluor 568-conjugated with anti-mouse IgG (both diluted 1:500; Molecular Probes, Invitrogen, Paisley, UK). For image acquisition we used a Leica TCS SP2 confocal microscope (Leica, Mannheim, Germany) equipped with an AOBS (acousto-optical beam splitter). Green fluorescence was visualized using an argon $488 \mathrm{~nm}$ laser with an emission bandwidth of $500-550 \mathrm{~nm}$. For red staining, an $\mathrm{He} / \mathrm{Ne} 543$ laser with an emission bandwidth of $600-730 \mathrm{~nm}$ was applied. Autofluorescence was also acquired using a 405-nm excitation laser with a $410-470 \mathrm{~nm}$ emission bandwidth.

\section{RESULTS}

\section{Clinical and biochemical investigations}

Proband from family A, II.1: a 42-year-old woman born to unrelated parents after an uncomplicated pregnancy. At the age of 32 years she was admitted with AKI of unknown origin. Exercise-induced renal failure was excluded and there was no need for dialysis. No urolithiasis was found. Renal biopsy was not performed. She suffered from frequent urinary tract infections in the subsequent years and received followed-up in nephrology outpatient department, where hypouricemia was found. Ultimately she was referred to our department for an unexplained finding of hypouricemia, where a detailed investigation of purine metabolism was performed. We have found hypouricemia $(0.9 \mathrm{mg} / \mathrm{dl}$, reference range: females, $2-5.7 \mathrm{mg} / \mathrm{dl}$; males, $2-7 \mathrm{mg} / \mathrm{dl}$ ) and an increased FE-UA (48\%, reference range: females, $7.3 \pm 1.3$; males, $10.3 \pm 4.2$ ) and normal excretion of uromoduline, xanthine and hypoxanthine.

Proband from family B, II.1: a 38-year-old male born to unrelated parents after an uncomplicated pregnancy. He had no significant medical history. His grandfather and aunt suffered from polycystic kidney disease. Linkage analysis in the examined family (maternal relatives) revealed the autosomal dominant polycystic kidney disease segregation with the PKD1 gene (OMIM no. 601313). No other family members had any obvious history of urolithiasis or exerciseinduced acute renal failure. As a fit, young man, he had biochemical tests performed as part of a routine wellness screen at age 26 years. The only abnormality was hypouricemia. This abnormal result of hypouricemia was found repeatedly $(0.5-0.7 \mathrm{mg} / \mathrm{dl})$. At age 38 years he was referred to our department for a detailed purine metabolic investigation. This revealed hypouricemia $(1.1 \mathrm{mg} / \mathrm{dl})$ with an increased FE-UA (43\%) and normal excretion of uromoduline, xanthine and hypoxanthine.

Proband from family C, II.2: a 5-year-old Czech male patient of Roma origin was recommended for metabolic investigation relating to progressive visual failure from age 3.5 years together with symptoms of progressive neurodegeneration. The data from patient's perinatal period were uneventful; the birth-weight was $3100 \mathrm{~g}$. As uricemia assessment is the common part of metabolic screening in patients with progressive neurologic symptomatology hypouricemia $(0.5-$ $0.8 \mathrm{mg} / \mathrm{dl}$ ) with an elevated FE-UA (39-52\%) was accidentally detected and subsequently confirmed. Excretion of uromoduline, xanthine and hypoxanthine were normal. No clinical or laboratory symptoms of renal disease were present in the patient. As hypouricemia did not explain the neurodegenerative disease the thorough examination continued and 10 months later the fatal neurometabolic 
disease, neuronal ceroid lipofuscinose type 7 (NCL7) conditioned by the homozygeous substitution in MFSD8 gene, typical for the Roma NCL patients from the middle Europe, was proved. ${ }^{25}$ Despite clarification of patient's condition, we decided to complete a hypouricemia examination. The patient's father did not live with family and was not available for examination. None of the patient's siblings suffered from NCL7. In accordance with results of the DNA analysis, prominent hypouricemia with the elevated FE-UA was found in the younger full brother of the patient (UA: $0.7 \mathrm{mg} / \mathrm{dl}$, FE-UA: $40 \%$ ) and in the younger full sister (UA: $0.5 \mathrm{mg} / \mathrm{dl}$, FE-UA: 57\%). The concentrations of UA in the mother and in two half-siblings were in the low-normal range, FE-UA values were on the border of the uppernormal range $(9 ; 13$; and $13 \%)$. All of them had normal excretion of uromoduline, xanthine and hypoxanthine. A comprehensive evaluation of personal histories and thorough laboratory examinations failed to find any signs of renal disease in either of the full siblings (II.3. II.4), the mother (I.1) or the older half-sibling (II.1). The younger half-sibling (II.5) underwent an acute urinary infection in the age of 1 month with a complete clinical and laboratory recovery after antibiotic treatment.

Clinical and biochemical data are shown in Table 1.

\section{Analysis of SLC22A12}

Decreased blood concentrations of UA together with markedly elevated FE-UA caused us to suspect RHUC and therefore an analysis of the SLC22A12 gene was performed after informed consent. DNA sequence variations found in the exon regions of the SLC22A12 gene of the probands are shown in Table 1.
Proband from family A, II.1: the heterozygous deletion c.1245_1253del (delGGCAGGGCT, p.L415_G417del) was found in exon 7. The heterozygous sequence variant c.1400C $>$ T (p.T467M) was found in exon 9. Cloning of the PCR product identified the genotype of proband family $\mathrm{A}$ as compound heterozygotes c.[1245_1253del $]+[1400 \mathrm{C}>\mathrm{T}]$. The same haplotype was found in sister II.2. The homozygous deletion c.1245_1253del in exon 7 was found in mother I.1.

Proband from family B, II.1: the heterozygous sequence variant c.1096G $>$ C (p.G366R) in exon 7 and previously published ${ }^{6}$ heterozygous sequence variant c.1430G $>$ A (p.R477H) in exon 9 were found. The cloning of the PCR product identified the genotype of proband family B as compound heterozygotes. c.[1096G $>$ C] + [1430G $>A$ ]. Heterozygous variant c.1096G $>C$ was found in mother I.2, heterozygous variant c.1430G $>$ A was found in father I. 1 and sister II.2.

Proband from family C, II.2: the homozygous deletion c.1245_1253del (delGGCAGGGCT, p.L415_G417del) was found in exon 7. The same haplotype was found in two full siblings (II.3, II.4). Heterozygous deletion was found in mother I.1 and two half-siblings (II.1, II.5).

Sequencing analysis found that none of the sequence variants were detected in a control cohort of 300 alleles (75 males, 75 females, randomly selected from patients at the General University Hospital, Prague. Moreover these sequence variants were also absent in 150 subjects from a majority Czech population with normouricemia (75 males, 75 females).

Two of three non-consanguineous probands with RHUC come from the minority Roma population. We collected a control cohort of 218 alleles from the Roma population in the Czech Republic and

Table 1 Clinical data and URAT1 allelic variants of the patients with renal hypouricemia

\begin{tabular}{|c|c|c|c|c|c|c|c|c|c|}
\hline & Family & $I D$ & $\begin{array}{l}\text { Year of } \\
\text { born }\end{array}$ & Sex & $\begin{array}{c}s-\cup A m g / d l r e f . r .: \\
2.0-5.7^{a} \\
2.0-7.0^{b}\end{array}$ & $\begin{array}{c}\text { FE-UA } \\
\text { \%ref. r.: } 7.3 \pm 1.3^{\mathrm{a}} \\
10.3 \pm 4.2^{\mathrm{b}}\end{array}$ & $\begin{array}{c}\text { s-creatinine } \mathrm{mg} / \mathrm{dl} r \mathrm{ref} \\
\text { r.: } 0.5-1.24^{\mathrm{a}} \\
0.5-1.1 \text { ד }\end{array}$ & Clinical symptoms & URAT1 \\
\hline 1. & $\begin{array}{c}\text { Family } \\
\text { A }\end{array}$ & I.1 & 1936 & $\mathrm{~F}$ & 2.1 & 52 & 1.5 & Renal failure (stage 1 ) & $\begin{array}{l}\text { p.[L415_G417del } \\
]+[\text { L415_G417del] }\end{array}$ \\
\hline 2. & & II.1 & 1970 & $\mathrm{~F}$ & $0.9-1.0$ & $48-53$ & $1.1-1.3$ & AKI & p.[L415_G417del] + [T467M]] \\
\hline 3. & & 11.2 & 1956 & $\mathrm{~F}$ & 1.3 & 60 & 1.7 & Asymptomatic & p.[L415_G417del] + [T467M] \\
\hline 4. & $\begin{array}{c}\text { Family } \\
\text { B }\end{array}$ & I.1 & 1946 & $\mathrm{~F}$ & 3.5 & 18 & 1.0 & Renal cysts (ADPKD) & p.[R477H] \\
\hline 5. & & 1.2 & 1943 & M & 3.5 & 12 & 1.1 & Asymptomatic & p.[G366R] \\
\hline 6. & & II.1 & 1974 & M & 1.1 & 43 & 1.1 & Renal cysts (ADPKD) & p. $[\mathrm{G} 366 \mathrm{R}]+[\mathrm{R} 477 \mathrm{H}]$ \\
\hline 7. & & 11.2 & 1979 & $\mathrm{~F}$ & 2.1 & 12 & 1.0 & Renal cysts (ADPKD) & p. $[\mathrm{R} 477 \mathrm{H}]$ \\
\hline 8. & $\begin{array}{l}\text { Family } \\
\text { C }\end{array}$ & I.1 & 1984 & $\mathrm{~F}$ & 2.5 & 13 & 0.7 & Asymptomatic & p.[L415_G417del] \\
\hline 9. & & $\begin{array}{c}11.1 \\
2003\end{array}$ & 2003 & M & 3.2 & 8,8 & 0.4 & Asymptomatic & p.[L415_G417del] \\
\hline 10. & & $\begin{array}{c}11.2 \\
2004\end{array}$ & 2004 & M & $0.5-0.8$ & $39-52$ & $0.4-0.6$ & $\begin{array}{l}\text { Visual failure and neurocognitive } \\
\text { deterioration since } 3.5 \text { years in } \\
\text { consequence of } \mathrm{NCL} 7\left(^{*}\right)\end{array}$ & $\begin{array}{l}\text { p.[L415_G417del ]+ } \\
{[\text { L415_G417del] }} \\
\text { *MFSD8 } \\
\text { p.[T294K]+ p.[T294K] }\end{array}$ \\
\hline 11. & & $\begin{array}{c}11.3 \\
2005\end{array}$ & 2005 & $\mathrm{~F}$ & 0.4 & 40 & 0.4 & Asymptomatic & $\begin{array}{l}\text { p.[L415_G417del } \\
]+[\text { L415_G417del] }\end{array}$ \\
\hline 12. & & $\begin{array}{c}11.4 \\
2006\end{array}$ & 2006 & M & 0.5 & 57 & 0.3 & Asymptomatic & $\begin{array}{l}\text { p.[L415_G417del } \\
]+[\text { L415_G417del] }\end{array}$ \\
\hline 13. & & $\begin{array}{c}11.5 \\
2009\end{array}$ & 2009 & M & 2.5 & NA & NA & Asymptomatic & p.[L415_G417del] \\
\hline
\end{tabular}

Abbreviations: ADPKD, autosomal dominant polycystic kidney disease; AKI, acute kidney injury; F, female; FE, fractional excretion; M, male; NA, not applicable; NCL, neuronal ceroid lipofuscinosis; ref. r., reference range; s, serum; UA, uric acid.

aReference range: childern under 15 years and female.

${ }^{b}$ Reference range: male. 
previously found sequence variants p.G366R, p.T467M and p.R477H were not detected. However, in two alleles, p.L415_G417del was detected in heterozygous status. Serum UA and FE-UA in these two subjects were within the reference range (9-year-old girl: $4.3 \mathrm{mg} / \mathrm{dl}$ and $18 \%$; 34-year-old male: $3.9 \mathrm{mg} / \mathrm{dl}$ and $8 \%$ ). These levels correspond with a previously described heterozygote for RHUC1. ${ }^{5}$ Finding two heterozygous control subjects confirmed an uneven geographical and ethnic distribution of SLC22A12 sequence variants.

\section{Functional studies of URAT1 variants}

The new missense mutations p.G366R, p.L415_G417del, p.T467M were tested for urate transport activity using in vitro expression analysis in Xenopus oocytes. The URAT1 p.R477H mutant and its urate-uptake activity was reported previously ${ }^{6}$ and examined here for comparison of its uptake ability relative to other mutants. As shown in Figure 1, the urate transport of URAT1 mutants, except p.R477H, were significantly decreased in comparison with the wt and were similar to that of oocytes that were not injected with URAT1 cRNA $\left({ }^{\star} P<0.05,{ }^{* *} P<0.01,{ }^{* *} P<0.001\right)$. This finding indicated that the above mentioned URAT1 mutations cause loss of urate reabsorption at the apical membrane of renal proximal tubules leading to decreased serum urate levels.

\section{Immunocytochemical analysis of URAT1 mutants}

To investigate the expression and plasma membrane targeting of URAT1 mutants, sections of mutant cRNA-injected oocytes stained with antiURAT1 polyclonal antibody were analyzed. Oocytes expressing the wt or p.R477H mutant exhibited strong continuous URAT1 immunostaining on the plasma membrane and disperse finely granular staining in the cytoplasm (Figure $2 \mathrm{~b}$ and c). Immunostaining of mutant's p.G366R and p.L415_G417del produced less intense staining, which was restricted to the cytoplasm, thus leaving the plasma membrane negative (Figure $2 \mathrm{~d}$ and e). Oocytes expressing p.T467M showed a weak discontinuous URAT1 signal on the plasma membrane and intracytoplasmic staining was lower than in the wt or p.R477H mutant (Figure 2f). Colocalization studies of URAT1 protein in the endoplasmic reticulum showed largely separate signals for wt, p.T467M and p.L415_G417del, however, in mutant's p.G366R and p.R477H the signals were fully or partially colocalized, respectively (Figure 3 ).

\section{DISCUSSION}

Recent data suggest a new concept of renal UA transport-multimolecular complex 'transportsome' that probably involves coopera-

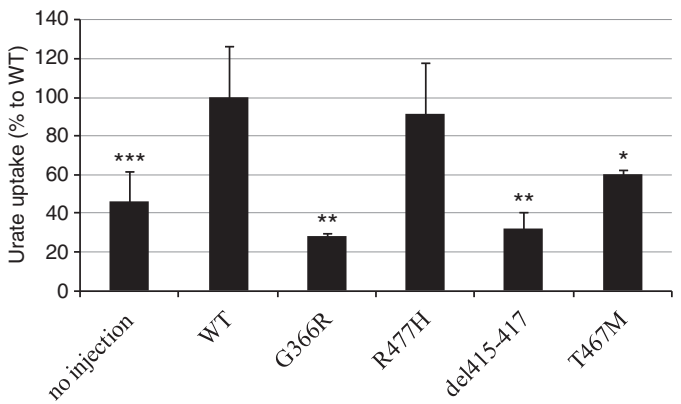

Figure 1 Urate transport ability of the URAT1 mutants found in the patients. Urate uptakes by the oocyte-expressed URAT1 mutants except p.R477H were significantly decreased compared with oocytes injected with wild-type (wt) URAT1 cRNA $\left({ }^{*} P<0.05, \quad * * P<0.01, \quad * * * P<0.001\right)$. p.R477H mutant showed the same urate-uptake ability as wt URAT1. No injection represents non-cRNA-injected oocytes. $n=6$ (no injection, wt) and 3 (p.G366R, p.R477H, p.del415-417, p.T467M). tion between multiple transporters. ${ }^{26}$ Further detailed studies concerning urate transporters and their interactions could clarify genotype/phenotype relationships in hyperuricemia and gout and also in conditions related to hyperuricemia-hypertension, cardiovascular diseases, insulin resistance and renal disorders.

RHUC was previously described in patients from Far and Middle East including Japan, Korea, China and Iraqi Jews. However, loss-offunction mutations affecting URAT1 has been shown in Caucasian population in Macedonia and in United Kingdom recently. ${ }^{15}$ Moreover, the case reports from European patients with symptomps of RHUC were reported in the past. ${ }^{27,28}$ In addition, three cases from Czech Republic and United Kingdom concerning patients with RHUC2 were also lately published. ${ }^{13,14}$ These reports and our findings suggest decreased awareness of RHUC outside of the Far East allows cases to go undetected. On the other hand, the high incidence of Far East RHUC is a reflection of the high allele frequency $(2.30-2.37 \%)$ of the p.W258X in SLC22A12 among Japanese and Koreans $^{6,7}$ indicating a founder mutation on Asian continent. Mutations described from European patients have not been shown as prevalent. However, our findings of two heterozygous in a control cohort of 109 subjects from the Roma population suggests the existence of prevalent variant p.L415_G417del in this minority population in the Czech Republic.

The structural model for URAT1 is mainly focused on the organization and alignment of residues within 12 transmembranespanning domains. ${ }^{3}$ Variant p.G366R and p.L415_G417del were present in equivalent positions within the membrane. Variants p.T467M and p.R477H were localized within the putative last extracellular loop. These variants, except the p.R477H, have not yet been identified in the SLC22A12 gene but the nature of this mutation strongly suggests that it is pathogenic; PolyPhen software (http:// genetics.bwh.harvard.edu/pph/) suggested that substitution p.G366R, p.T467M and p.R477H were probably damaging. Moreover, other predictive software, SIFT (http://sift.jcvi.org/), suggests that p.G366R and p.R477H are damaging with high confidence predictions. Although variant p.T467M is tolerated with a sift score of 0.3 . The prediction of the possible impact of the $9 \mathrm{bp}$ deletion p.L415_G417del, three amino acid deletion, on the structure and function of a human protein by in silico analysis did not suggest a structure-function relationship.

The results of in silico analysis did not fit into the results of the functional study. Variants p.R477H had urate uptake comparable with the wt protein. Furthermore, immunocytochemical analysis exhibited strong continuous URAT1 immunostaining of the plasma membrane and dispersed fine granular staining in the cytoplasm. On other hand, p.G366R, p.L415_G417del and p.T467M were significantly decreased in comparison with the wt. URAT1 signal on the plasma membrane was absent in mutants p.G366R and p.L415_G417del and significantly reduced in mutant p.T467M. Colocalization studies showed accumulation of URAT1 protein in the endoplasmic reticulum of p.G366R mutant. This suggests that synthesized mutant protein do not reach the plasma membrane. This conclusion suggested that part of URAT1 deficiencies could be based on mechanism of protein misfolding.

In silico analysis is insufficient for evidence of causality relative to sequence variants except for nonsense mutations resulting in premature stop codons and putative mutant proteins. Evolutionary analysis of URAT1 paralogs, including five mammalian species and Xenopus tropicalis, revealed high conservation of all mutated amino acids (Figure 4). The possible explanation of the neutral effect of allelic variant p.R477H is that both amino acids are basic. On the 

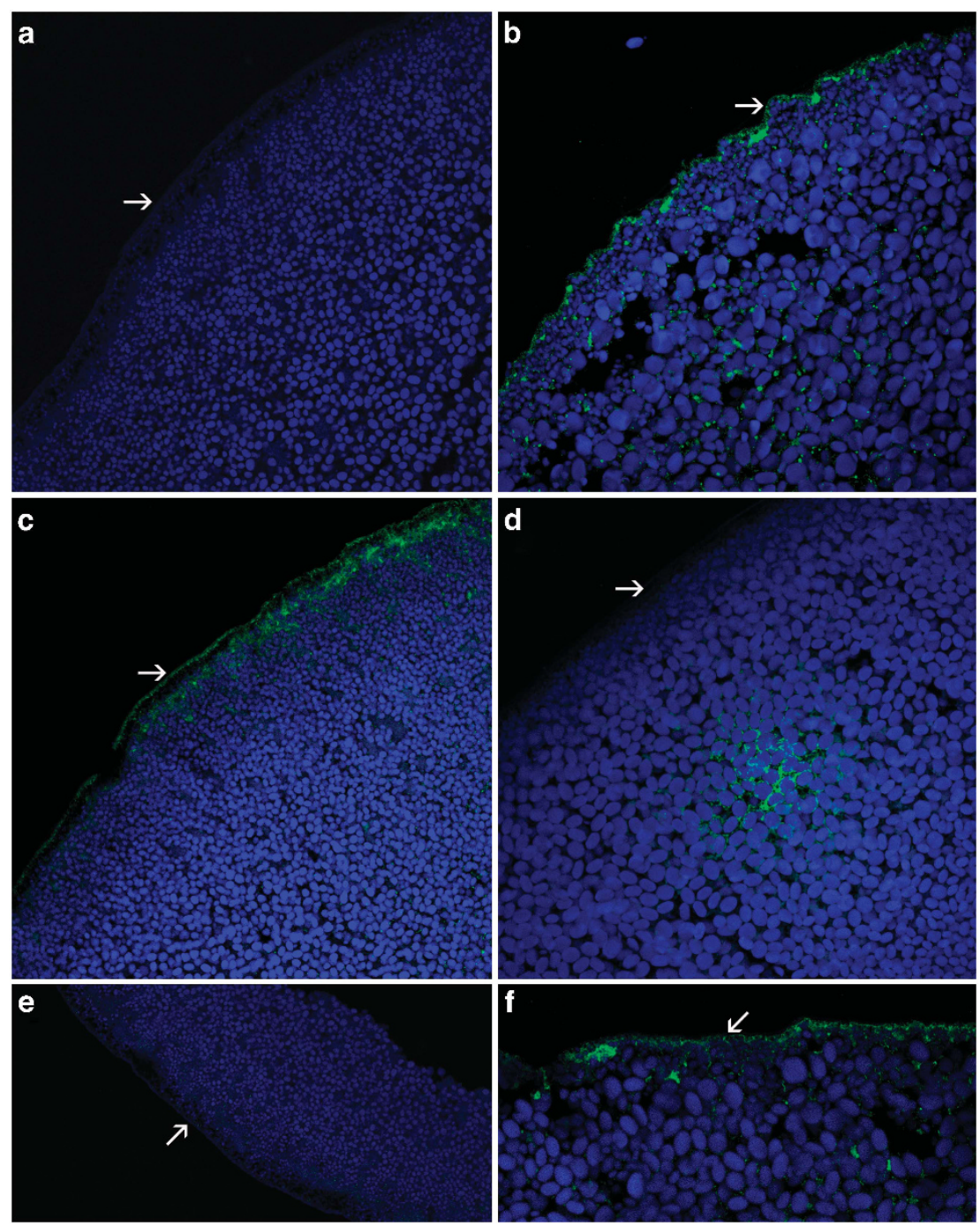

Figure 2 Immunocytochemical analysis of oocytes injected with $50 \mathrm{ng}$ of cRNA encoding the wild-type (wt) or mutant URAT1 performed with anti-URAT1 polyclonal antibody. The signal of URAT1 is green, autofluorescent granules in cytoplasm of oocytes give a blue signal. (a) Water-injected oocyte without any detectable URAT1 signal. (b, c) Oocytes injected with the wt cRNA (b) or with CRNA of p.R477H (c) exhibited a strong linear signal on the plasma membrane and a finely granular intracytoplasmic signal. (d, e) The mutant p.G366R (d) and p.L415_G417del (e) were characterized by the lack of plasma membrane expression of URAT1. Cytoplasmic staining intensity was lower than in the wt or p.R477H. Note the tendency of intracytoplasmic signal accumulation in p.G366R (d). Staining of mutant p.T467M was faint and irregular on the plasma membrane and reduced in cytoplasm (f). Plasma membrane of oocytes is marked by arrows.

other hand the remaining two substitutions significantly changed local chemical and physical attributes. In p.G366R, non-polar glycine is substituted for the positively charged basic arginine and polar threonine, while in p.T467M the change involves non-polar methionine. The contradictory conclusions from the in silico survey and function study need to be elucidated by further characterization of novel sequence variants for evidence of causality in RHUC.

The phenotypic severity of RHUC1 is not correlated with results of functional characterization of URAT1 variants. The genotype c. $\left[1245 \_1253 \mathrm{del}\right]+[1400 \mathrm{C}>\mathrm{T}]$ was found in the proband from family A and her sister. Although habits, life style, clinical investigations, including biochemical levels of serum UA, fractional excretion of UA and creatinine in serum, were approximately the same in both siblings, the proband from family A repetitively suffers AKI. However, her sister is without clinical symptoms of urolithiasis and/or AKI. The same homozygote genotype c.[1245_1253del] was found in the mother of the proband from family A with renal failure and three siblings from family $\mathrm{C}$ were without clinical symptoms. Normal levels of serum UA $(124 \mu \mathrm{mol} / \mathrm{l})$ in the mother of the family A proband was probably caused by obesity, her body mass index was $>35$. Although AKI in patient A I.1 did not appear, FE-UA was constantly high ( $>50 \%)$ and she had slightly reduced kidney function (stage 1) with serum creatinine $110 \mu \mathrm{mol} / \mathrm{l}$ (reference range: females, $0.5-1.24 \mathrm{mg} / \mathrm{dl}$; males, $0.5-1.17 \mathrm{mg} / \mathrm{dl}$ ). However, the absence of renal complications in family $\mathrm{C}$ was likely caused by their young age. Detection of hyperuricemia in family $\mathrm{C}$ was accidental. Multiple homozygosity for ethnically prevalent mutations are typical in closely related populations with high levels of inbreeding. This is particularly true in middle Europe especially in some Roma communities. As the course of neurodegenerative disease in the double homozygote for NCL7 and RHUC1 did not differ significantly from other Roma patients with the same genotype in the MFSD8 gene, it appears that hypouricemia 


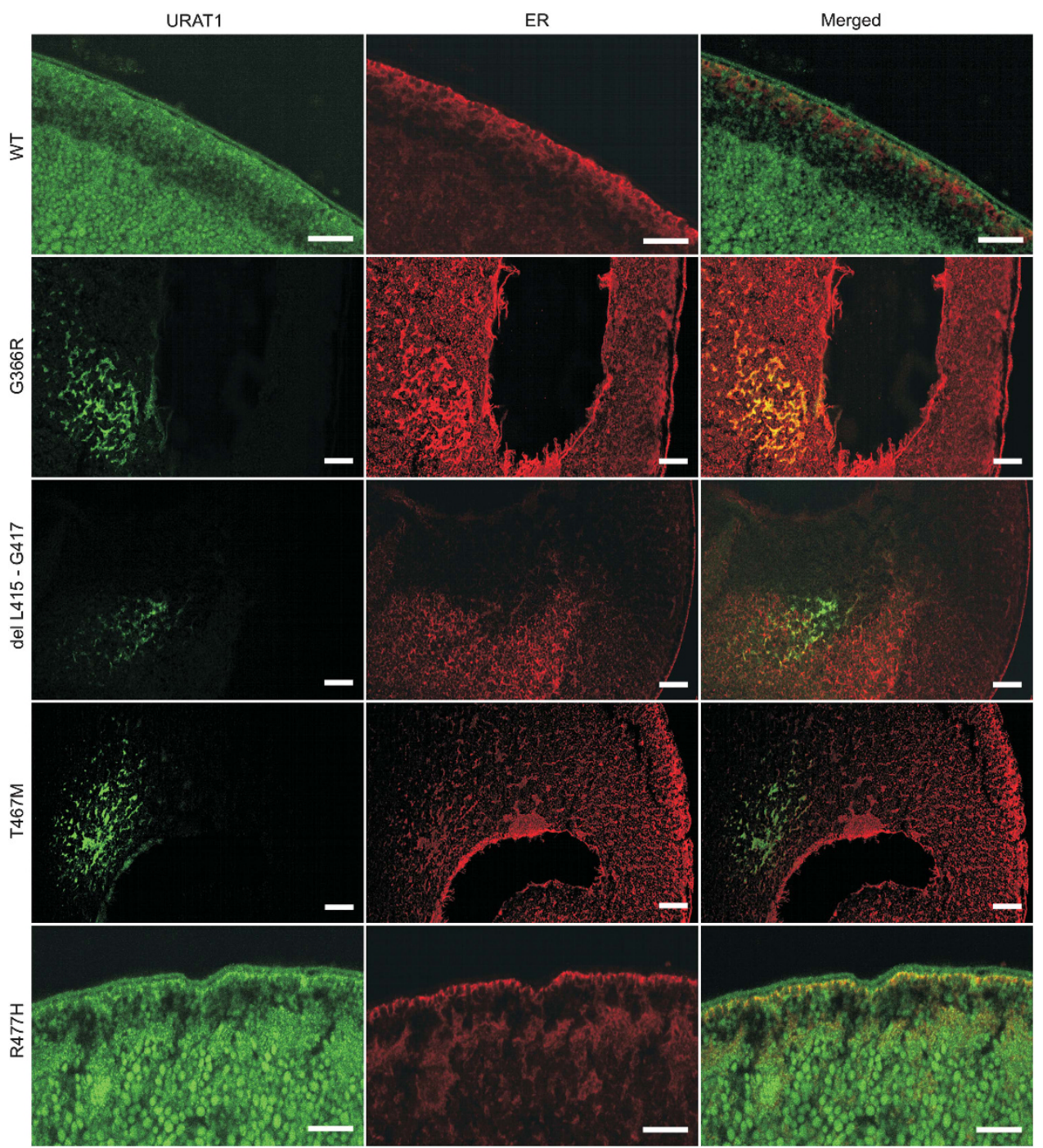

Figure 3 Colocalization of URAT1 mutant variants with endoplasmatic reticulum (ER). Strong colocalization with ER is observed in p.G366R. URAT1 localization in wild-type (wt) and p.R477H is predominantly in cytoplasmic membrane. Dispersed signal is also seen in cytoplasm. Partial colocalization with ER is visible in p.R477H mutant. In wt the URAT1 and ER signals are rather separated. Scale bar represents $50 \mu \mathrm{m}$.

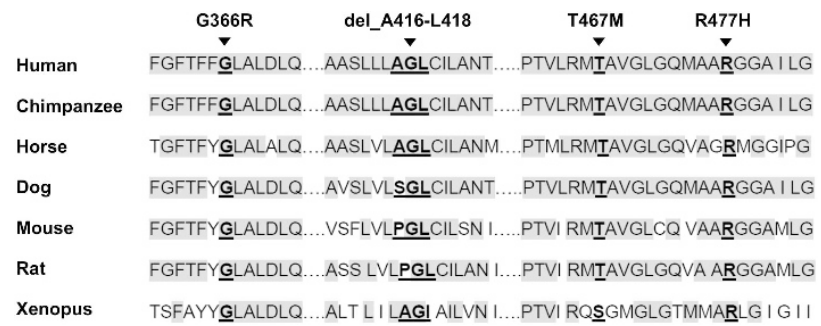

Figure 4 URAT1 allelic variants. Alignment of the URAT1 amino acids in the studied allelic variants with chimpanzee, horse, dog, mouse, rat and Xenopus paralogs.

does not influence ongoing primary neurodegeneration in the mentioned combination of the 2 non-cosegregated monogenic disorders (loci 4q28.2 and 11q13.1).

Observation of neurological symptoms in hypouricemia patients might provide new information concerning the relationship between UA and neurological diseases. UA is a powerful scavenger of peroxide radicals, hydroxide radicals and singlet oxygen in human biological fluids. ${ }^{29,30}$ UA accounts for up to $60 \%$ of plasma antioxidative capacity and presumably protects not only erythrocytes, but also DNA-contained in long-lived $\mathrm{T}$ and $\mathrm{B}$ lymphocytes and macrophages. UA may also protect other tissues as they are, presumably, in equilibrium with blood UA. ${ }^{29}$ UA has been hypothesized to protect against oxidative stress, a prominent contributor to dopaminergic neuron degeneration in Parkinson's disease. Studies have evaluated a potential association between serum UA and the risk of developing Parkinson's disease, finding a lower risk among individuals with higher levels of serum UA. ${ }^{31,32}$ Significant associations were detected between variations in SLC2A9 and memory performance. It was observed that higher UA levels might be associated with increased performance on memory-related tasks. ${ }^{33}$ However, in patients with RHUC, neurological symptoms were not observed-including cases with extremely low serum UA (values near 0 ).

RHUC has clinical variability and only about $10 \%$ of all patients with a defect in SLC22A12 gene have nephrolithiasis and/or AKI. A defect in the SLC2A9 gene results in more patients with renal failure. 
AKI in RHUC2 was reported in three homozygous probands ${ }^{11}$ and three compound heterozygous ${ }^{12,14}$ from a sample of 15 patients and was not described in patients with a heterozygous mutation. These investigations suggest that the frequency of AKI might be different between RHUC1 and heterozygous and homozygous RHUC2. Various mechanisms have been proposed to explain how RHUC causes AKI. UA nephropathy could result from an increase in UA production during exercise. ${ }^{34}$ Renal reperfusion injury, due to vasoconstriction, results from an exercise-induced increase in oxygen free radicals potentiated by a lack of UA. ${ }^{35}$ Inactivation of urate/anion transporters eliminate UA absorption and also block secretion of organic anions into the tubular lumen with toxic effects on the renal proximal tubules leading to toxic acute tubular cell injury. ${ }^{11}$

In conclusion, RHUC is a rare disorder, but it also needs to be considered in patients coming from areas other than Asia. It is obvious, that for the reliable genotype-phenotype relations, more patients need to be analyzed. Urate concentration in serum and urine may provide an initial indication of urate transporter deficiency before sequencing analysis is performed. The key to identifying the disorder is greater awareness among primary care physicians, nephrologists and urologists of urate transporter deficiency. The diagnosis and therapeutic management requires a multidisciplinary approach.

\section{CONFLICT OF INTEREST}

The authors declare no conflict of interest.

\section{ACKNOWLEDGEMENTS}

This study was supported by a grant from the Czech Republic, Ministry of Health (IGA MZ NT/11322-4/2010) and, partly, by Institutional support PRVOUK-P24/LF1/3 program of the Charles University in Prague. The authors thank Lenka Hornova for the clinical data for proband A, Petr Vyletal for analysis of uromodulin and Makoto Hosoyamada for training related to functional studies.

1 Watanabe S, Kang DH, Feng L et al: Uric acid, hominoid evolution, and the pathogenesis of salt-sensitivity. Hypertension 2002; 40: 355-360.

2 Sotgiu S, Pugliatti M, Sanna A et al: Serum uric acid and multiple sclerosis. Neurol Sci 2002; 23: 183-188.

3 Enomoto A, Kimura H, Chairoungdua A et al: Molecular identification of a renal urateanion exchanger that regulates blood urate levels. Nature 2002; 417: 447-452.

4 Augustin R, Carayannopoulos MO, Dowd L, Phay JE, Moley JF, Moley KH: Identification and characterization of human glucose transporter-like protein-9 (GLUT9): alternative splicing alters trafficking. J Biol Chem 2004; 279: 16229-16236.

5 Ichida K, Hosoyamada M, Hisatome I et al: Clinical and molecular analysis of patients with renal hypouricemia in Japan-influence of URAT1 gene on urinary urate excretion. J Am Soc Nephrol 2004; 15: 164-173.

6 Iwai N, Mino Y, Hosoyamada M, Tago N, Kokubo Y, Endou H: A high prevalence of renal hypouricemia caused by inactive SLC22A12 in Japanese. Kidney Int 2004; 66: 935-944.

7 Ichida K, Hosoyamada M, Kamatani N et al: Age and origin of the G774A mutation in SLC22A12 causing renal hypouricemia in Japanese. Clin Genet 2008; 74: 243-251.

8 Taniguchi A, Urano W, Yamanaka $\mathrm{M}$ et al: A common mutation in an organic anion transporter gene, SLC22A12, is a suppressing factor for the development of gout. Arthritis Rheum 2005; 52: 2576-2577.
9 Matsuo H, Chiba T, Nagamori S et al: Mutations in glucose transporter 9 gene SLC2A9 cause renal hypouricemia. Am J Hum Genet 2008; 83: 744-751.

10 Anzai $\mathrm{N}$, Ichida $\mathrm{K}$, Jutabha $\mathrm{P}$ et al: Plasma urate level is directly regulated by a voltagedriven urate efflux transporter URATv1 (SLC2A9) in humans. J Biol Chem 2008; 283: 26834-26838.

11 Dinour D, Gray NK, Campbell S et al: Homozygous SLC2A9 mutations cause severe renal hypouricemia. J Am Soc Nephrol 2010; 21: 64-72.

12 Shima $Y$, Nozu K, Nozu $Y$ et al: Recurrent EIARF and PRES with severe renal hypouricemia by compound heterozygous SLC2A9 mutation. Pediatrics 2011; 127: e1621-e1625

13 Stiburkova B, Ichida K, Sebesta I: Novel homozygous insertion in SLC2A9 gene caused renal hypouricemia. Mol Genet Metab 2011; 102: 430-435.

14 Stiburkova B, Taylor J, Marinaki AM, Sebesta I: Acute kidney injury in two children caused by renal hypouricaemia type 2. Pediatr Nephrol 2012; 27: 1411-1415.

15 Tasic V, Hynes AM, Kitamura $\mathrm{K}$ et al: Clinical and Functional Characterization of URAT1 Variants. PLoS One 2011; 6: e28641.

16 Lam CW, Kong AP, Tsui TK et al: A novel mutation of SLC22A12 gene causing primary renal hypouricemia in a patient with metabolic syndrome. Clin Chim Acta 2008; 398: $157-158$.

17 Dinour D, Bahn A, Ganon L et al: URAT1 mutations cause renal hypouricemia type 1 in Iraqi Jews. Nephrol Dial Transplant 2011; 26: 2175-2181.

18 Wakida N, Tuyen DG, Adachi M et al: Mutations in human urate transporter 1 gene in presecretory reabsorption defect type of familial renal hypouricemia. J Clin Endocrino Metab 2005; 90: 2169-2174.

19 Vázquez-Mellado J, Jiménez-Vaca AL, Cuevas-Covarrubias S, Alvarado-Romano V, Pozo-Molina G, Burgos-Vargas R: Molecular analysis of the SLC22A12 (URAT1) gene in patients with primary gout. Rheumatology 2007; 46: 215-219.

20 Tin A, Woodward OM, Kao WHL et al: Genome-wide association study for serum urate concentrations and gout among African Americans identifies genomic risk loci and a novel URAT1 loss-of-function allele. Hum Mol Genet 2011; 20: 4056-4068.

21 Graessler J, Graessler A, Unger S et al: Association of the human urate transporter 1 with reduced renal uric acid excretion and hyperuricemia in a German Caucasian population. Arthritis Rheum 2006; 54: 292-300.

22 Tabara Y, Kohara K, Kawamoto R et al: Association of four genetic loci with uric acid levels and reduced renal function: The J-SHIPP Suita Study. Am J Nephrol 2010; 32: 279-286.

23 Stiburkova B, Krijt J, Vyletal P et al: Novel mutations in xanthine dehydrogenase/ oxidase cause severe hypouricemia: biochemical and molecular genetic analysis in two Czech families with xanthinuria type I. Clin Chim Acta 2012; 413: 93-99.

24 Nakamura M, Anzai N, Jutabha P, Sato H, Sakurai H, Ichida K: Concentrationdependent inhibitory effect of irbesartan on renal uric acid transporters. J Pharmacol Sci 2010; 114: 115-118.

25 Kousi M, Siintola E, Dvorakova L et al: Mutations in CLN7/MFSD8 are a common cause of variant late-infantile neuronal ceroid lipofuscinosis. Brain 2009; 132 : 810-819.

26 Anzai $\mathrm{N}$, Kanai $\mathrm{Y}$, Endou $\mathrm{H}$ : New insights into renal transport of urate. Curr Opin Rheumatol 2007; 19: 151-157.

27 Erley CM, Hirschberg RR, Hoefer W, Schaefer K: Acute renal failure due to uric acid nephropathy in a patient with renal hypouricemia. Klin Wochenschr 1989; 67: 308-312.

28 Ouellet G, Lin SH, Nolin L, Bonnardeaux A: Hereditary renal hypouricemia in a Caucasian patient: a case report and review of the literature. Nephrol Ther 2009; 5: 568-571.

29 Ames BN, Cathcart R, Schwiers E, Hochstein P. Uric acid provides an antioxidant defense in humans against oxidant- and radical-caused aging and cancer: a hypothesis. Proc Natl Acad Sci USA 1981; 78: 6858-6862.

30 Glantzounis GK, Tsimoyiannis EC, Kappas AM, Galaris DA: Uric acid and oxidative stress. Curr Pharm Des 2005; 11: 4145-4151.

31 Davis JW, Grandinetti A, Waslien CI, Ross GW, White LR, Morens DM: Observations on serum uric acid levels and the risk of idiopathic Parkinson's disease. Am J Epidemiol 1996; 144: 480-484.

32 de Lau LM, Koudstaal PJ, Hofman A, Breteler MM: Serum uric acid levels and the risk of Parkinson disease. Ann Neurol 2005; 58: 797-800.

33 Houlihan LM, Wyatt ND, Harris SE et al: Variation in the uric acid transporter gene (SLC2A9) and memory performance. Hum Mol Genet 2010; 19: 2321-2330.

34 Bell DW, Yeung RS, Bohlander SK, Cheng JQ, Jin F, Testa JR: A microdissection library of the rat renal carcinoma gene region. Cytogenet Cell Genet 1995; 70: 92-94.

35 Naitoh M, Suzuki H, Murakami $M$ et al: Arginine vasopressin produces renal vasodilation via V2 receptors in conscious dogs. Am J Physiol 1993; 265: 934-942. 